\section{BAS/ HISTONE DEACETYLASE 3 PROTECTS ENDOTHELIAL CELLS BSCR2 6 FROM INFLAMMATION VIA REGULATION OF GALECTIN 9 EXPRESSION}

doi:10.1136/hrt.2010.205781.37

Saydul Alam, Hongling Li, Daniel Martin, Anna Zampetaki, Yanhua Hu, Qingbo Xu, Lingfang Zeng. Cardiovascular Division, King's College London BHFCentre, London, UK

Histone deacetylase 3 (HDAC3), a member of the class I histone deacetylases, is known to have a crucial role in endothelial cell differentiation ${ }^{1} 2$ and maintenance of endothelial integrity in response to disturbed flow. ${ }^{3}$ In this study, we investigated the function of HDAC3 in endothelial protection from inflammation, and the underlying mechanism. The inflammatory mediator lipopolysaccharides (LPS) induced HDAC3 and galectin-9 production in a similar pattern in human umbilical vein endothelial cells. Overexpression of HDAC3 by adenoviral gene transfer increased galectin- 9 expression. Pharmacological inhibition of HDAC activity with a panHDAC inhibitor (TSA) or HDAC3 specific inhibitor (apicidin) reduced the baseline and LPS-induced galectin- 9 expression. In addition, knockdown of HDAC3 through shRNA lentiviral transfection abolished the baseline and LPS-induced galectin-9 expression. Similar results were observed on interferon $\gamma$ (IFN $\gamma$ )-induced galectin-9 expression. To explore the underlying mechanism, the interaction of HDAC3 with galectin-9 upstream signal pathway phosphoinositol-3kinase (PI3K)/signal transmission and transducer 3 (Stat3)/interferon response factor (IRF3) was assessed. Co-immunoprecipitation assay showed that HDAC3 formed a complex with PI3K/Stat3/IRF3, which was enhanced by LPS and IFN $\gamma$ treatment. Using truncated forms of HDAC3, it was shown that the C-terminal of HDAC3 was responsible for the formation of the complex. Furthermore, venous administration of LPS or IFN $\gamma$ to mice increased HDAC3 expression and binding to the above-mentioned proteins, leading to galectin- 9 expression in the aorta. These results suggest that HDAC3 may protect endothelial cell from inflammation through galectin-9 expression, which may have an impact on preventing vascular inflammation related to the development of atherosclerosis.

\section{REFERENCES}

1. Zeng L, Xiao Q, Margariti A, Zhang Z, et al. HDAC3 is crucial in shear- and VEGFinduced stem cell differentiation toward endothelial cells. J Cell Biol 2006; 174(7):1059-69

2. Xiao $\mathbf{0}$, Zeng $L$, Zhang $Z$, et al. Sca-1+ progenitors derived from embryonic stem cells differentiate into endothelial cells capable of vascular repair after arterial injury. Arterioscler Thromb Vasc Biol 2006;26(10):2244-51.

3. Zampetaki A, Zeng L, Margariti A, et al. Histone deacetylase 3 is critical in endothelial survival and atherosclerosis development in response to disturbed flow. Circulation 2010;121(1):132-42. Epub 2009 Dec 21.

\section{BAS/ DIESEL EXHAUST PARTICLES PROMOTE ATHEROSCLEROSIS BSCR27 IN APOLIPOPROTEIN E-DEFICIENT MICE}

doi:10.1136/hrt.2010.205781.38

${ }^{1} \mathrm{M}$ R Miller, ${ }^{1} \mathrm{~S}$ G McLean, ${ }^{2} \mathrm{R}$ Duffin, ${ }^{1} \mathrm{C}$ A Shaw, ${ }^{1} \mathrm{~N}$ L Mills, ${ }^{2} \mathrm{~K}$ Donaldson, ${ }^{1} \mathrm{D}$ E Newby, ${ }^{1} \mathrm{P}$ W F Hadoke. ${ }^{1}$ Centre of Cardiovascular Science, University of Edinburgh, Edinburgh, UK; ${ }^{2}$ Centre for Inflammation Research, University of Edinburgh, Edinburgh, UK

Air pollution has been linked to the development of atherosclerosis and cardiovascular disease. Diesel exhaust particulate (DEP) accounts for a substantial proportion of urban air pollution but its effects on atherogenesis are unknown. We hypothesised that DEP will exacerbate plaque formation in a murine model of atherosclerosis.

Apolipoprotein E knockout (ApoE) mice (10-12 weeks; $n=16$ ) were fed a 'Western diet' (21\% cholesterol) for 8 weeks to induce the development of 'complex' atherosclerotic plaques. During the last 4 weeks of feeding, mice underwent twice-weekly intratracheal instillation of $35 \mu \mathrm{l} \mathrm{DEP}(1 \mathrm{mg} / \mathrm{ml}$; National Institute of Standards and Technology) or vehicle (saline).
Histological sections of the brachiocephalic artery from ApoE knockout mice showed large, foam cell-filled fibrous plaques. Plaque burden was increased $(\mathrm{p}=0.025 ; \mathrm{n}=5-6)$ in DEP-treated mice

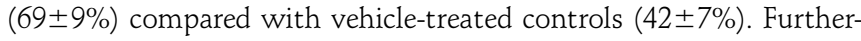
more, plaques from DEP-treated mice exhibited a greater number of adjoining $(2.3 \pm 0.2 \%)$ and buried $(1.2 \pm 0.3 \%)$ fibrous caps than control mice $(1.7 \pm 0.2 \%$ and $0.2 \pm 0.1 \%$, respectively; $p<0.05 n=5)$. There was no evidence of systemic inflammation, increased circulating blood lipids or endothelial dysfunction in DEP-treated animals.

This is the first study to show that pulmonary exposure to the particulate matter within diesel exhaust enhances atherogenesis. This action may, therefore, contribute to the increased cardiovascular morbidity and mortality associated with air pollution. This model will allow identification of the constituents of DEP that mediate this atherogenic effect and provide an important insight into potential interventions to reduce the impact of vehicular emissions.

\section{BAS/ BLOCKADE OF ADENOSINE A2A RECEPTOR ATTENUATES BSCR28 ANGIOTENSIN II-INDUCED REACTIVE OXYGEN SPECIES PRODUCTION AND IMPAIRMENT OF ENDOTHELIUM- DEPENDENT VESSEL RELAXATION IN MOUSE AORTAS}

doi:10.1136/hrt.2010.205781.39

Junjie Du, Susanna Hourani, Jian-Mei Li. Faculty of Health and Medical Sciences, University of Surrey, Guildford, Surrey, UK

The endothelium expresses abundantly an adenosine A2A receptor (A2AR) which has important roles in the regulation of vascular function. The endothelium expresses also the AT1-type receptor for angiotensin II (AngII) which has been found to activate a reactive oxygen species (ROS)-generating NADPH oxidase and cause oxidative damage to the endothelium. However, little is known about the role of A2AR in AngII-induced endothelial ROS production. In this study, we investigated the effect of A2AR blockade on AngIIinduced ROS production and endothelium function using freshly isolated aortas from CD1 mice at 10-12 weeks of age. Compared with vessels treated with vehicle, acute AngII (200 nM for $45 \mathrm{~min}$ ) treatment significantly increased ROS production in the vessel wall as detected by DHE fluorescence and this was accompanied by increased ERK1/2 phosphorylation. These AngII effects were inhibited, returning to control levels in the presence of a specific A2AR antagonist, SCH58261 (100 nM). Compared with control vessels, treatment with AngII severely compromised the endothelium-dependent vessel relaxation to acetylcholine as assessed by an organ bath. Addition of SCH58261 (100 nM) or tiron $(20 \mathrm{mM}$, a specific cell membrane permeable superoxide scavenger) during AngII stimulation protected the endothelium from AngII damage and preserved endothelium-dependent vessel relaxation to acetylcholine. The endothelium dependence of the relaxation to acetylcholine was confirmed by mechanical denudation of the endothelium. In conclusion, blockade of A2AR protects the endothelium from acute AngII-induced oxidative stress, ERK1/2 phosphorylation and endothelium dysfunction.

\section{BAS/ APOLIPOPROTEIN(A) IMPAIRS ADAPTIVE REMODELLING IN BSCR29 HUMAN SAPHENOUS VEIN ENDOTHELIAL AND SMOOTH MUSCLE CELLS}

doi:10.1136/hrt.2010.205781.40

${ }^{1} \mathrm{~K}$ Riches, ${ }^{1} \mathrm{~L}$ Franklin, ${ }^{1} \mathrm{R}$ Chowdhury, ${ }^{1} \mathrm{~A}$ Magbool, ${ }^{1} \mathrm{D} \mathrm{J}$ O'Regan, ${ }^{1} \mathrm{~S}$ G Ball, ${ }^{2} \mathrm{M}$ L Koschinsky, ${ }^{1} \mathrm{~N}$ A Turner, ${ }^{1} \mathrm{~K}$ E Porter. ${ }^{1}$ Division of Cardiovascular \& Neuronal Remodelling, LIGHT, University of Leeds, Leeds UK; ${ }^{2}$ Department of Biochemistry, University of Windsor, Windsor, Ontario, Canada

Apolipoprotein(a) (apo(a)), a unique glycoprotein component of plasma lipoprotein(a) (Lp(a)),exhibits resistance to classical 